\title{
Association Between Obstructive Sleep Apnea- Hypopnea Syndrome and Outcomes in Patients with Myocardial Infarction in the Absence of Obstructive Coronary Artery Disease
}

\section{Chaojie He}

First Hospital of Jiaxing

\section{Linfeng Cao}

First Hospital of Jiaxing

Chunyan Zhu

First Hospital of Jiaxing

\section{Yujuan Zhu}

First Hospital of Jiaxing

\section{Shijun Wang}

First Hospital of Jiaxing

Changlin Zhai

First Hospital of Jiaxing

Hui-lin Hu ( $\square$ huhuilin1979@sohu.com )

First Hospital of Jiaxing https://orcid.org/0000-0003-1619-4682

\section{Research article}

Keywords: obstructive sleep apnea-hypopnea syndrome, coronary artery disease, outcome

Posted Date: May 7th, 2020

DOI: https://doi.org/10.21203/rs.3.rs-26413/v1

License: (9) (i) This work is licensed under a Creative Commons Attribution 4.0 International License. Read Full License

Version of Record: A version of this preprint was published at Frontiers in Cardiovascular Medicine on October 23rd, 2020. See the published version at https://doi.org/10.3389/fcvm.2020.573819. 


\section{Abstract}

Background: Myocardial infarction in the absence of obstructive coronary artery disease (MINOCA) occurs in $5 \%$ to $10 \%$ of all patients with acute myocardial infarction. Obstructive sleep apnea-hypopnea syndrome (OSAHS) is linked to increased cardiovascular morbidity and mortality, but the relationship of OSAHS and outcomes in patients with MINOCA remains unknown.

Objectives: We aimed to evaluate the association between OSAHS and clinical outcomes in patients with MINOCA.

Methods and Results: Between January 2015 and December 2016, we carried out a prospective and consecutive cohort study of 583 patients with MINOCA and followed them up for three years. An apneahypopnea index of $\geq 15$ events per hour recorded by polysomnography was defined as the diagnostic criterion for OSAHS. The primary end point was all-cause mortality, and the second end point was major adverse cardiovascular or cerebrovascular events (MACCE), a composite of cardiac death, nonfatal myocardial infarction, heart failure, cardiovascular-related rehospitalization, and stroke. All-cause mortality happened in 69 patients and MACCE occurred in 113 patients during the three-year follow-up. Kaplan-Meier survival curves indicated the significant relationship of OSAHS with all-cause mortality (log-rank $P=0.012$ ) and MACCE (log-rank $P=0.002)$. Multivariate Cox regression analysis indicated OSAHS as an independent predictor of all-cause mortality and MACCE (adjusted hazard ratio: 1.714; 95\% confidence interval $(\mathrm{Cl}): 1.203-2.168 ; \mathrm{P}=0.013$; and adjusted hazard ratio: 1.787; 95\% Cl: 1.249-2.236; $\mathrm{P}$ $=0.004$; respectively), independent of age, sex, cardiovascular risk factors and discharge medications.

Conclusions: OSAHS is independently associated with increased risk of all-cause mortality and MACCE in patients with MINOCA. Intervention and treatment should be considered to alleviate OSAHS-associated risk.

\section{Introduction}

Myocardial infarction in the absence of obstructive coronary artery disease (MINOCA) is a distinct entity with multiple causes defined by acute presentation of myocardial infarction with no remarkable stenosis of coronary artery on coronary angiography (stenosis $<50 \%$ ). ${ }^{1-3}$ In June 2019 , the American Heart Association (AHA) has systematically described recommendations for contemporary diagnosis and management of MINOCA, and they have broadened our horizon to understand this heterogeneous disease. ${ }^{4}$ More than 660000 percutaneous coronary intervention ( $\mathrm{PCl}$ ) procedures were performed in China in 2017, and approximately $10 \%$ of emergency coronary angiography results showed the absence of evident obstructive coronary stenosis. ${ }^{5}$ Although the number of initial diagnosis of MINOCA is considerable owing to the huge population and demand for $\mathrm{PCl}$, the clinical outcomes and follow-up of MINOCA have received minimal attention with the result indicating a portion of individuals may not receive appropriate treatment. ${ }^{6-7}$ 
Sleep apnea-hypopnea syndrome (SAHS) is a syndrome of nocturnal respiratory interruptions resulting in snoring, sleep fragmentation, and daytime drowsiness, with a prevalence ranging from $5.7-17 \%$ in adults. ${ }^{8-10}$ Obstructive SAHS is the most common manifestation of sleep apnea and it is caused by nocturnal upper airway collapse with paradoxical thoracoabdominal movement. ${ }^{8}$ In several multi-center registries, obstructive sleep apnea was observed in $45.3-65.7 \%$ of patients with myocardial infarction or who underwent $\mathrm{PCl}{ }^{11,12}$ Accumulating evidence has demonstrated that OSAHS is an emerging risk factor for arteriosclerosis, coronary heart disease, hypertension, stroke, cardiovascular rhythm, and heart

failure (HF). ${ }^{13-17}$ Numerous studies have shown that continuous positive airway pressure (CPAP) therapy could retard the progression of coronary artery disease (CAD) and decrease the rate of occurrence of cardiovascular mortality, stroke, and nonfatal myocardial infarction. ${ }^{18-21}$

To our best knowledge, no published data are currently available concerning the prevalence of OSAHS in patients with MINOCA. In addition, the association between OSAHS and clinical outcomes has not been determined in this population. This study aimed to investigate the prevalence of OSAHS in patients with MINOCA and to evaluate the relationship between OSAHS and clinical outcomes in patients with MINOCA.

\section{Methods}

\section{Study design and population}

Our study complied with the Declaration of Helsinki and was approved by the Ethics Committee (The First Affiliated Hospital of Jiaxing University, Jiaxing, China). All participants provided their informed written consent before being enrolled in the present study.

A total of 583 patients who had been referred to First Affiliated Hospital of Jiaxing University for emergency coronary angiography and diagnosed with MINOCA were included consecutively in the present study between January 2015 and December 2016. Jiaxing University Hospital has a Chest Pain Center that covers a $4000 \mathrm{sq}$. $\mathrm{km}$ area and serves about six million residents. MINOCA was diagnosed with the following criteria in line with a position paper of European Society on cardiology: (1) the diagnostic criteria involving the Fourth Universal Definition of Myocardial Infarction criteria; (2) coronary angiography with no artery lesions $\geq 50 \%$ in any infarct-related artery; (3) no other clinically overt specific cause that can explain acute presentation. ${ }^{22}$ The exclusion criteria were extremely strict in the present study: (1) mimic myocardial infarction, such as sepsis, pulmonary embolism, cardiac contusion, overlooked obstructive CAD, coronary emboli/thrombus, Takotsubo syndrome, and myocarditis; ${ }^{4}$ (2)cognitive disorder or mental confusion; (3) known OSAHS on CPAP therapy; (4) patients' refusal to give a written informed consent. The patients were divided in two groups, namely, the OSAHS and non-OSAHS groups, in accordance with the evaluation of OSAHS.

\section{Data collection}


We used inpatient record system to collect the data on demography, laboratory, electrocardiogram, echocardiography, coronary angiography, and discharge medication. Data collectors were blinded to the study group assignment. After discharge, all patients were followed-up for three years by physicians in First Affiliated Hospital of Jiaxing University. Follow-up was performed by outpatient clinic visit, patient record system, telephone contact or instant messaging (WeChat). In case of failure to contact the patients, we used their family members' contact information and asked patients to visit the outpatient clinic annually. All individuals were advised to contact us immediately if they experience angina or uncomfortable chest stuffiness.

\section{Diagnosis of OSAHS}

All enrolled patients were required to complete the Berlin Questionnaire. ${ }^{23}$ The Chinese version of Berlin Questionnaire is a convenient and inexpensive self-assessment method, which consists of 10 questions in three categories. The questionnaire assesses the presence of sleep apnea by persistent symptoms of snoring, the severity of daytime sleepiness, and a history of hypertension or a body mass index (BMI) greater than $30 \mathrm{~kg} / \mathrm{m}^{2}$. A positive score in at least two out of three categories indicates a high risk of OSAHS. $^{23}$

All individuals were asked to monitor their overnight sleep by using a polysomnography device (SOMNOmedics GmbH \& Co. KG, Germany) with the assistance of pneumology department regardless of their results on the Berlin Questionnaire. Polysomnography is the golden standard for obstructive sleep apnea diagnosis. The apnea-hypopnea index $(\mathrm{AHI})$ was quantified as the average number of apnea and/or hypopnea per hour of the total recorded time. Apnea was defined by a $90 \%$ or greater reduction in airflow lasting at least $10 \mathrm{~s}$. Hypopnea was defined as a $30 \%$ or greater drop in respiratory airflow lasting $\geq 10 \mathrm{~s}$ accompanied with an oxygen desaturation $\geq 4 \% .{ }^{24}$ Obstructive sleep apnea was defined as the absence of airflow in the presence of paradoxical thoracoabdominal movement. An AHI of $>$ or $=15$ events/h was considered prospectively as the criterion for OSAHS (AHI values of $5-15,15-30$ and $>30$ indicate mild, moderate and severe symptoms, respectively). ${ }^{24}$

\section{Primary and secondary end points}

The primary end point in the present study was all-cause mortality. The secondary end point was a major adverse cardiovascular or cerebrovascular events (MACCE), defined as a composite of cardiac death, nonfatal myocardial infarction, HF. cardiovascular-related rehospitalization, and stroke. Cardiac death refers to death with evidence of acute coronary syndrome, cardiac arrhythmia or congestive HF. The diagnosis of stroke was performed in line with the AHA guidelines for the scientific statement of stroke. ${ }^{25}$ The definition of HF was determined in accordance with the current guidelines published by European Society of Cardiology. ${ }^{26}$ Cardiovascular-related rehospitalization means any cardiovascular causes for readmission. Mortality and MACCE were first verified by searching the patient record system by means of HaiTai software. Death certificates were obtained for any patients who died during the follow-up period if available. All individuals were then contacted by telephone or instant messaging (WeChat) for 
assessment of end points. End point confirmations were conducted by two physicians without any knowledge of diagnosis of OSAHS.

\section{Statistical analysis}

Data were analyzed with the SPSS version 19.0 software (IBM, SPSS Inc., Chicago, IL). Continuous variables were presented as the mean and standard deviation and categorical variables were expressed as frequencies and percentages. Differences between means were analyzed with Student $t$ test. Differences between proportions were assessed with Chi-square test or Fisher's exact test as appropriate. Kaplan-Meier curves of the survival function were calculated for patients with OSAHS and non-OSAHS, and the two groups were compared with the log-rank test. Multiple Cox proportional hazards model was used to identify prognostic factors after adjusting for potential confounders significantly associated with the outcome in the univariate analysis at a level of $P<0.20$. The proportional hazards assumption was evaluated for Cox regression. The Fine and Gray's models were used to assess the risk of MACE where allcause mortality was considered as competing event. Results of Cox regression are presented as hazard ratio (HR) with $95 \%$ confidence interval $(\mathrm{Cl})$. A two-tailed $\mathrm{P}$ values $<0.05$ was considered statistically significant.

\section{Results}

\section{Baseline characteristics of patients}

The study objects included 730 patients with acute myocardial infarction and nonobstructive coronary arteries. A total of 147 patients were excluded from the present trial: 2 with cognitive disorder, 3 with sepsis, 4 with pulmonary embolism, 2 with overlooked obstructive CAD, 4 with coronary emboli/thrombus, 33 with Takotsubo syndrome, 67 with myocarditis, 15 patients on CPAP therapy, and 17 patients who refused to participate in the study. This prospective cohort study recruited 583 patients with MINOCA (Fig. 1), of which 8 individuals terminated study participation or were lost to follow-up. The Berlin Questionnaires of 237 patients have indicated a high risk of sleep apnea and/or hypopnea, and 158 patients were diagnosed with OSAHS by polysomnography, with a prevalence of $27.1 \%$. Whereas, only $70.9 \%$ of the patients in the OSAHS group were classified as high risk by the Berlin Questionnaire. Patient baseline characteristics are listed in Table 1. Compared with the control group, OSAHS patients had a higher proportion of males and a history of smoking but without further significant differences regarding age, BMI, cardiovascular risk factors, medications at discharge, laboratory, electrocardiogram, echocardiography, and coronary angiography data. Table 2 shows the sleep results from OSAHS and non-OSAHS groups. Of 158 OSAHS individuals, 84 presented moderate symptoms, and 74 exhibited severe ones. The mean AHI values were $29.0 \pm 12.2$ and $5.3 \pm 4.2$ for OSAHS and non-OSAHS groups, respectively. The mean lowest SpO2 values during apnea were 82.7 \pm 7.9 and $91.3 \pm 4.1$, respectively. 15 patients with moderate or severe OSAHS agreed to receive CPAP treatment. The present study was to investigate the association between untreated OSHAS and clinical outcomes in patients with MINOCA, 
patients who had received CPAP therapy for more than one month during the study were excluded from analysis. 
Table 1

Baseline characteristics of the study population with or without OSAHS

\section{Characteristics}

With OSAHS $(n=158)$

Demographics

Age, mean $\pm S D, y$

$64.5 \pm 12.9$

$63.4 \pm 12.7$

0.158

Female, $\mathrm{n}(\%)$

$62(39.2)$

$24.9 \pm 3.6$

$268(63.1)$

$\llbracket 0.001$

$B M I$, mean $\pm S D, k g / m^{2}$

Risk factors, $\mathrm{n}(\%)$

Smoking

$52(32.9)$

88 (55.7)

$36(22.8)$

19 (12.0)

9 (5.7)

$8(5.1)$

Heart failure

Medications at discharge, $\mathrm{n}(\%)$

Anti-platelets

135 (85.4)

$96(60.8)$

79(50.0)

146 (92.4)

97 (22.8)

0.013

BBlockers

RAAS inhibitors

Statins

Electrocardiographic changes on admission, $\mathrm{n}(\%)$

STEMI

$20(12.7)$

$138(87.3)$

NSTEMI

$201(47.3)$

0.071

84 (19.7)

0.423

$38(8.9)$

0.265

$15(3.5)$

0.242

$19(4.5)$

0.762

Echocardiography, mean \pm SD

LVEF (\%)

$54.3 \pm 13.4$

$54.9 \pm 13.5$

0.715

Laboratory parameters on admission, mean \pm SD

Pro-BNP $(\mathrm{pg} / \mathrm{mL})$

$986.4 \pm 1423.3$

$919 \pm 1356.3$

0.778

$\mathrm{cTnT}(\mathrm{ng} / \mathrm{mL})$

$2.1 \pm 1.0$

$2.0 \pm 0.9$

0.765

OSAHS indicates obstructive sleep apnea-hypopnea syndrome; SD, standard deviation; BMI, body mass index; COPD, chronic obstructive pulmonary disease; RAAS, rennin-angiotensin-aldosterone system; STEMI, ST-segment elevation myocardial infarction; NSTEMI, non-ST-segment elevation myocardial infarction; LVEF; left ventricular ejection fraction; Pro-BNP, pro-brain natriuretic peptide; cTnT, cardiac troponin T; CRP, C reactive protein. 


\begin{tabular}{|c|c|c|c|}
\hline Characteristics & With OSAHS $(n=158)$ & Without OSAHS $(n=425)$ & $P$ value \\
\hline CRP (mg/L) & $15.6 \pm 4.7$ & $15.7 \pm 5.1$ & 0.756 \\
\hline \multicolumn{4}{|c|}{ Angiographic data, $\mathrm{n}(\%)$} \\
\hline Normal vessels & $18(11.4)$ & $40(9.4)$ & 0.478 \\
\hline Stenosis $\leq 30 \%$ & $72(45.6)$ & $202(47.5)$ & 0.673 \\
\hline $30 \% \bigotimes$ Stenosis $₫ 50 \%$ & $68(43.0)$ & $183(43.1)$ & 0.996 \\
\hline \multicolumn{4}{|c|}{$\begin{array}{l}\text { OSAHS indicates obstructive sleep apnea-hypopnea syndrome; SD, standard deviation; BMI, body } \\
\text { mass index; COPD, chronic obstructive pulmonary disease; RAAS, rennin-angiotensin-aldosterone } \\
\text { system; STEMI, ST-segment elevation myocardial infarction; NSTEMI, non-ST-segment elevation } \\
\text { myocardial infarction; LVEF; left ventricular ejection fraction; Pro-BNP, pro-brain natriuretic peptide; } \\
\text { cTnT, cardiac troponin T; CRP, C reactive protein. }\end{array}$} \\
\hline
\end{tabular}

Table 2

The Berlin Questionnaire results and sleep respiratory events recorded by polysomnography

\begin{tabular}{|llll|}
\hline Characteristics & With OSAHS $(\mathbf{n}=\mathbf{1 5 8})$ & Without OSAHS $(\mathbf{n}=\mathbf{4 2 5})$ & P value \\
\hline AHI, mean \pm SD, /h & $29.0 \pm 12.2$ & $5.3 \pm 4.2$ & $\varangle 0.001$ \\
\hline Baseline SpO2,mean \pm SD, \% & $94.3 \pm 4.1$ & $95.0 \pm 3.9$ & 0.349 \\
\hline Lowest SpO2,mean \pm SD, \% & $82.7 \pm 7.9$ & $91.3 \pm 4.1$ & $\varangle 0.001$ \\
\hline High-risk BQ, $n(\%)$ & $112(70.9)$ & $125(29.4)$ & $\varangle 0.001$ \\
\hline $\begin{array}{l}\text { OSAHS indicates obstructive sleep apnea-hypopnea syndrome; AHI, apnea hypopnea index; SD, } \\
\text { standard deviation; BQ, Berlin Questionnaire. }\end{array}$ & & \\
\hline
\end{tabular}

\section{Medications}

Medications prescribed at hospital discharge are presented in Table 1. No significant differences were observed between the OSAHS group and non-OSAHS group in the use of medications at discharge. On the basis of prescription refill at the latest follow-up, $89.4 \%$ of the patients in the OSHAS group and $91.2 \%$ of the patients in the non-OSAHS group have insisted on antiplatelet therapy. Besides, a total of $89.6 \%$ and $87.4 \%$ of patients in the OSHAS group were adherent to statin and beta-blocker therapy, compared with $87.9 \%$ and $86.8 \%$ in the non-OSAHS group, respectively.

\section{Follow-up and clinical outcomes medications}

A total of 69 all-cause mortality and 113 MACCE events occurred during the three-year observation period in both groups. MACCE included cardiac death in 51 patients (45.1\%), nonfatal myocardial infarction in 11 (9.7\%), HF in 4 (3.5\%), cardiovascular-related rehospitalization in $24(21.2 \%)$ and stroke in $23(20.6 \%)$. The Kaplan-Meier cumulative survival curves indicated a significant association of OSAHS with allcause mortality (log-rank $\mathrm{P}=0.012$ ) and MACCE (log-rank $\mathrm{P}=0.002$ ) (Figs. 2 and 3 , respectively). In univariate Cox regression analysis, OSAHS predicted the incidence of all-cause mortality and MACCE 
(unadjusted HR = 1.805: 95\% Cl: 1.113-2.928; $\mathrm{P}=0.017$; and HR: $1.811 ; 95 \% \mathrm{Cl}: 1.237-2.650 ; \mathrm{P}=0.002$; respectively). After adjustment for age, sex, cardiovascular risk factors, and medications in multivariate Cox regression analysis, OSAHS remained an independent predictor of all-cause mortality and MACCE (adjusted HR: 1.714; 95\% Cl: 1.203-2.168; P = 0.013; and adjusted HR: 1.787; 95\% Cl: 1.249-2.236; P = 0.004; respectively). The results are summarized in Tables 3 and 4. Notably, rennin-angiotensinaldosterone system (RAAS) inhibitors, statins, and ST-segment elevation myocardial infarction (STEMI) showed a significant correlation with clinical outcomes, agreeing with the findings of previous studies. ${ }^{32}$

Table 3

Multivariate Cox regression of variables influencing All-cause mortality

\begin{tabular}{|lll|}
\hline Variables & HR $(95 \%$ Cl $)$ & P value \\
\hline Age & $1.014(0.956-1.107)$ & 0.756 \\
\hline Female & $0.851(0.599-1.103)$ & 0.308 \\
\hline Smoking & $1.196(0.825-1.524)$ & 0.269 \\
\hline COPD & $1.095(0.796-1.397)$ & 0.553 \\
\hline Hypertension & $1.112(0.795-1.299)$ & 0.694 \\
\hline Diabetes & $1.169(0.869-1.403)$ & 0.463 \\
\hline Heart failure & $1.306(0.869-1.823)$ & 0.118 \\
\hline Anti-platelets & $0.845(0.702-1.190)$ & 0.756 \\
\hline$\beta$-Blockers & $0.774(0.491-1.203)$ & 0.365 \\
\hline RAAS inhibitors & $0.697(0.429-0.989)$ & 0.031 \\
\hline Statins & $0.507(0.299-0.679)$ & $<0.001$ \\
\hline STEMI & $3.813(1.192-9.243)$ & $<0.001$ \\
\hline OSAHS & $1.714(1.203-2.168)$ & 0.013 \\
\hline $\begin{array}{l}\text { HR indicates hazard ratio; COPD, chronic obstructive pulmonary disease; RAAS, rennin-angiotensin- } \\
\text { aldosterone system; STEMI, ST-segment elevation myocardial infarction; OSAHS, obstructive sleep } \\
\text { apnea-hypopnea syndrome. }\end{array}$ & \\
\hline
\end{tabular}


Table 4

Multivariate Cox regression of variables influencing MACCE

\begin{tabular}{|lll|}
\hline Variables & HR $(95 \%$ Cl $)$ & P value \\
\hline Age & $1.026(0.946-1.089)$ & 0.823 \\
\hline Female & $0.906(0.772-1.165)$ & 0.364 \\
\hline Smoking & $1.121(0.889-1.342)$ & 0.471 \\
\hline COPD & $1.162(0.912-1.446)$ & 0.456 \\
\hline Hypertension & $1.094(0.803-1.303)$ & 0.761 \\
\hline Diabetes & $1.119(0.892-1.563)$ & 0.429 \\
\hline Heart failure & $1.386(0.877-1.806)$ & 0.095 \\
\hline Anti-platelets & $0.846(0.621-1.110)$ & 0.469 \\
\hline B-Blockers & $0.689(0.592-1.099)$ & 0.112 \\
\hline RAAS inhibitors & $0.624(0.412-0.941)$ & $<0.01$ \\
\hline Statins & $0.476(0.299-0.776)$ & $<0.001$ \\
\hline STEMI & $4.633(1.956-10.041)$ & $<0.001$ \\
\hline OSAHS & $1.787(1.249-2.236)$ & 0.004 \\
\hline $\begin{array}{l}\text { MACCE indicates major adverse cardiovascular or cerebrovascular events; HR, hazard ratio; COPD, } \\
\text { chronic obstructive pulmonary disease; RAAS, rennin-angiotensin-aldosterone system; STEMI, ST- } \\
\text { segment elevation myocardial infarction; OSAHS, obstructive sleep apnea-hypopnea syndrome. }\end{array}$ \\
\hline
\end{tabular}

\section{Discussion}

To our knowledge, this research is the first study to explore the prevalence of OSAHS in patients with MINOCA and demonstrate the possible relationship between OSAHS and clinical outcomes in this population. In this cohort of 583 patients with MINOCA, the prevalence of OSAHS was $27.1 \%$, similar to other CAD population. We observed that OSAHS was associated with an increased risk of all-cause mortality and MACCE comprising cardiac death, nonfatal myocardial infarction, HF, cardiovascularrelated rehospitalization, and stroke. After adjusting for demographics, cardiovascular risk factors, and discharge medications, patients with OSAHS exhibited 1.714 times risk of all-cause mortality and 1.787 times risk of MACCE at three-year follow-up.

The previous large multi-center registry, The Sleep and Stent Study ${ }^{11}$ has shown that obstructive sleep apnea is independently associated with cardiovascular adverse events in patients with CAD, with the prevalence reaching $45.3 \%$. A similar study conducted by Lee also has reported that sleep apnea prevalence is as high as $65.7 \%$ in patients admitted with AMI. ${ }^{12}$ However, the prevalence of OSAHS in patients with MINOCA is unknown. Although the identical diagnostic criterion of OSAHS was an AHI $\geq 15$ 
in the present study, OSAHS was found in $27.1 \%$ of patients with MINOCA, which is relatively lower compared with that of CAD patients described by previous studies. In addition, we observed that only $70.9 \%$ of the individuals with OSAHS were classified as high risk by the Berlin Questionnaire. The results suggested that the sensitivity and specificity of Berlin Questionnaire remained controversial.

OSAHS is characterized by intermittent upper airway collapse with paradoxical thoracoabdominal movement during sleep, resulting in biochemical and haemodynamic disorders, including sympathetic activation, increased platelet agreeability, hypercoagulability, ultra-inflammation responses, endothelial dysfunction, increased pulmonary vascular resistance, and increased left ventricular load caused by decrease in intrathoracic pressure. ${ }^{27-31}$ These potential mechanisms together contribute to the progression of atherosclerosis, myocardial infarction, hypertension, arrhythmia, HF, and stroke. ${ }^{8}$ In the study of Mooe et al., the co-existence of CAD and sleep apnea resulted in 70\% relative increase and a $10.7 \%$ absolute increase in the composite of death, cerebrovascular events, and myocardial infarction after a median period of 5.1-year follow-up. ${ }^{32}$ In one study, obstructive sleep apnea was demonstrated as an independent predictor for clinical outcomes in patients with acute coronary syndrome after $\mathrm{PCl} .{ }^{33} \mathrm{The}$ main finding of our observational study is that OSAHS is independently associated with all-cause mortality and MACCE in patients with MINOCA. The total-cause mortality of MINOCA in the three-year follow-up was $11.8 \%$ in the present study, agreeing with the SWEDEHEART registry, with a mortality of $13.4 \%$ after mean follow-up of 4.1 years. ${ }^{34}$ OSAHS patients had an adjusted OR of 1.787 for the MACCE when compared with patients without sleep apnea. Interestingly, we observed that stroke accounted for one-fifth of MACCE in the current study, 16 in patients with OSAHS and 7 in the control group, which is consistent with previous findings indicating OSAHS as an independent risk factor for stroke. ${ }^{13,35-36}$

The design of present study has certain strengths. First, all patients with OSAHS were diagnosed with gold standard by supervised polysomnography during hospitalization. Second, we strictly excluded clinically overt causes for a myocardial injury, such as myocarditis, sepsis, pulmonary embolism, Takotsubo syndrome, an potentially overlooked obstructive CAD from the current study, consistent with the clinical algorithm for the recently introduced diagnosis of MINOCA by AHA. ${ }^{4}$ Thus, the definition of MINOCA utilized in the current study is in accordance with the statement of contemporary diagnosis and management of patients with MINOCA, thereby further supporting our results. Third, the well-known confounding risk and preventive factors that affect clinical outcomes in patients with MINOCA, such as traditional cardiovascular risk factors, STEMI, RAAS inhibitors, and statins, were all analyzed for adjustment. Fourthly, the rate of loss to follow-up was low in the present study owing to the convenient instant messaging app (WeChat).

Several limitations need to be considered in the present study. First, the present study is an observational single Chest Pain Center study, and the results may not be generalizable. Second, the sample size of the current study is relatively small due to the critical inclusion and exclusion criteria. Third, the exclusion of patients who refused to participate in the trial and loss of follow-up could contribute to a bias of the results in present study. 


\section{Conclusion}

OSAHS is common among patients with MINOCA and is independently associated with increased risk of all-cause mortality and MACCE in patients with MINOCA. The results highlight the importance of management in this population with OSAHS. Intervention and treatment should be considered to alleviate OSAHS-associated risks. Further studies are warranted to determine the effectiveness of CPAP treatment in MINOCA population.

\section{Declarations}

\section{Declaration of conflicting interests}

The authors declared no potential conflicts of interest with respect to this article.

\section{Funding}

None.

\section{Acknowledgements}

The authors gratefully acknowledge the patients and volunteers who made this study possible.

\section{CRediT author statement}

He Chaojie: Conceptualization, Methodology, Investigation, Writing-Original Draft. Cao Linfeng: Data curation, Formal analysis, Writing- Original draft preparation. Zhu Chunyan: Resourses,Visualization, Investigation. Zhu Yujuan: Formal analysis, Validation, Resourses. Wang Shijun: Software, Validation. Zhai Changlin: Project administration, Writing- Reviewing and Editing, Hu Huilin: Conceptualization, Methodology, Writing- Reviewing and Editing, Supervision.

\section{References}

1. Pasupathy S, Air T, Dreyer RP, et al. Systematic review of patients presenting with suspected myocardial infarction and nonobstructive coronary arteries. Circulation. 2015;131:861-70.

2. Poku N, Noble S. Myocardial infarction with non obstructive coronary arteries (MINOCA): a whole new ball game. Expert Rev Cardiovasc Ther. 2017;15:7-14.

3. Vidal-Perez R. Abou Jokh Casas C, Agra-Bermejo RM, et al. Myocardial infarction with nonobstructive coronary arteries: A comprehensive review and future research directions. World $\mathrm{J}$ Cardiol 2019; 11: 305-15. 
4. Tamis-Holland JE, Jneid H, Reynolds HR, et al. Contemporary Diagnosis and Management of Patients With Myocardial Infarction in the Absence of Obstructive Coronary Artery Disease: A Scientific Statement From the American Heart Association. Circulation. 2019;139:e891-908.

5. Abdu FA. Liu L, Mohammed AQ, et al. Myocardial infarction with non-obstructive coronary arteries (MINOCA) in Chinese patients: Clinical features, treatment and 1 year follow-up. Int J Cardiol 2019; 287: 27-31.

6. Jánosi A. Ferenci T, Kőszegi Z, et al. Myocardial infarction without obstructive coronary artery disease (MINOCA) - prevalence and prognosis. Orv Hetil 2019; 160: 1791-7.

7. Buono A. Pedrotti $P$, Soriano $F$, et al. Myocardial infarction with non-obstructive coronary arteries (MINOCA): diagnosis, pathogenesis, therapy and prognosis. G Ital Cardiol (Rome) 2019; 20: 499511.

8. Jaffe LM. Kjekshus J, Gottlieb SS. Importance and management of chronic sleep apnoea in cardiology. Eur Heart J 2013; 34: 809-15.

9. Young T. Palta M, Dempsey J, et al. The occurrence of sleep-disordered breathing among middleaged adults. N Engl J Med 1993; 328: 1230-5.

10. Hauri PJ,editor. The international Classification of Sleep Disorders, Diagnostic and Coding Manual. 2nd ed.. Westchester: American Academy of Sleep Medicine; 2005.

11. Lee CH. Sethi R, Li R, et al. Obstructive Sleep Apnea and Cardiovascular Events After Percutaneous Coronary Intervention. Circulation 2016; 133: 2008-17.

12. Lee $\mathrm{CH}$. Khoo SM, Tai BC, et al. Obstructive Sleep Apnea in Patients Admitted for Acute Myocardial Infarction. Prevalence, Predictors, and Effect on Microvascular Perfusion. Chest 2009; 135: 1488-95.

13. Yaggi HK. Concato J, Kernan WN, et al. Obstructive Sleep Apnea as a Risk Factor for Stroke and Death. N Engl J Med 2005; 353: 2034-41.

14. Meng F. Ma JH, Wang W, et al. Obstructive Sleep Apnea Syndrome Is a Risk Factor of Hypertension. Minerva Med 2016; 107: 294-9.

15. Gami AS. Hodge DO, Herges RM, et al. Obstructive sleep apnea, obesity, and the risk of incident atrial fibrillation. J Am Coll Cardiol 2007; 49: 565-71.

16. Javaheri S. Sleep disorders in systolic heart failure: a prospective study of 100 male patients. The final report. Int J Cardiol. 2006;106:21-8.

17. Savransky V. Nanayakkara A, Li J, et al. Chronic intermittent hypoxia induces atherosclerosis. Am J Respir Crit Care Med 2007; 175: 1290-7.

18. Buchner NJ. Sanner BM, Borgel J, et al. Continuous Positive Airway Pressure Treatment of Mild to Moderate Obstructive Sleep Apnea Reduces Cardiovascular Risk. Am J Respir Crit Care Med 2007; 176: $1274-80$.

19. Wang X. Zhang Y, Dong ZM, et al. Effect of Continuous Positive Airway Pressure on Long-Term Cardiovascular Outcomes in Patients With Coronary Artery Disease and Obstructive Sleep Apnea: A Systematic Review and Meta-Analysis. Respir Res 2018; 19: 61. 
20. Yu J. Zhou Z, McEvoy RD, et al. Association of Positive Airway Pressure With Cardiovascular Events and Death in Adults With Sleep Apnea: A Systematic Review and Meta-analysis. JAMA 2017; 318: $156-66$.

21. Kim Y. Koo YS, Lee HY, et al. Can Continuous Positive Airway Pressure Reduce the Risk of Stroke in Obstructive Sleep Apnea Patients? A Systematic Review and Meta-Analysis. PLoS One 2016; 11: e0146317.

22. Agewall S, Beltrame JF, Reynolds HR, et al. ESC working group position paper on myocardial infarction with non-obstructive coronary arteries. Eur Heart J. 2017;38:143-53.

23. Netzer NC. Stoohs RA, Netzer CM, et al. Using the Berlin Questionnaire to identify patients at risk for the sleep apnea syndrome. Ann Intern Med 1999; 131: 485-91.

24. Iber C. Ancoli-Israel S, Chesson AL, et al. AASM manual for the scoring of sleep and associated events: rules, terminology and technical specification. Westchester: American Academy of Sleep Medicine, 2007.

25. Sacco RL. Kasner SE, Broderick JP, et al. An updated definition of stroke for the 21st century: a statement for healthcare professionals from the American Heart Association/American Stroke Association. Stroke 2013; 44: 2064-89.

26. Ponikowski P. Voors AA, Anker SD, et al. 2016 ESC Guidelines for the diagnosis and treatment of acute and chronic heart failure: The Task Force for the diagnosis and treatment of acute and chronic heart failure of the European Society of Cardiology (ESC)Developed with the special contribution of the Heart Failure Association (HFA) of the ESC. Eur Heart J 2016;37: 2129-200.

27. Bonsignore MR. Marrone 0 , Insalaco G, et al. The cardiovascular effects of obstructive sleep apnoeas: analysis of pathogenic mechanisms. Eur Respir J 1994; 7: 786-805.

28. Somers VK. Mark AL, Zavala DC, et al. Contrasting effects of hypoxia and hypercapnia on ventilation and sympathetic activity in humans. J Appl Physiol 1989; 67: 2101-6.

29. Bradley TD. Floras JS. Obstructive sleep apnoea and its cardiovascular consequences. Lancet. 2009; 373: 82-93.

30. Golbin JM. Somers VK, Caples SM. Obstructive sleep apnea, cardiovascular disease, and pulmonary hypertension. Proc Am Thorac Soc 2008; 5: 200-6.

31. Jiang XM. Qian XS, Gao XF, et al. Obstructive Sleep Apnea Affecting Platelet Reactivity in Patients Undergoing Percutaneous Coronary Intervention. Chin Med J (Engl) 2018; 131: 1023-9.

32. Mooe T. Franklin KA, Holmstro"m K, et al. Sleep-disordered breathing and coronary artery disease: long-term prognosis. Am J Respir Crit Care Med 2001; 164: 1910-3.

33. Yumino D. Tsurumi Y, Takagi A,et al. Impact of obstructive sleep apnea on clinical and angiographic outcomes following percutaneous coronary intervention in patients with acute coronary syndrome. Am J Cardiol 2007; 99: 26-30.

34. Lindahl B. Baron T, Erlinge D, et al. Medical Therapy for Secondary Prevention and Long-Term Outcome in Patients With Myocardial Infarction With Nonobstructive Coronary Artery Disease. Circulation 2017; 135: 1481-9. 
35. King S. Cuellar N. Obstructive Sleep Apnea as an Independent Stroke Risk Factor: A Review of the Evidence, Stroke Prevention Guidelines, and Implications for Neuroscience Nursing Practice. J Neurosci Nurs 2016; 48: 133-42.

36. Godoy J. Mellado P, Tapia J, et al. Obstructive Sleep Apnea as an Independent Stroke Risk Factor: Possible Mechanisms. Curr Mol Med 2009; 9: 203-9.

\section{Figures}

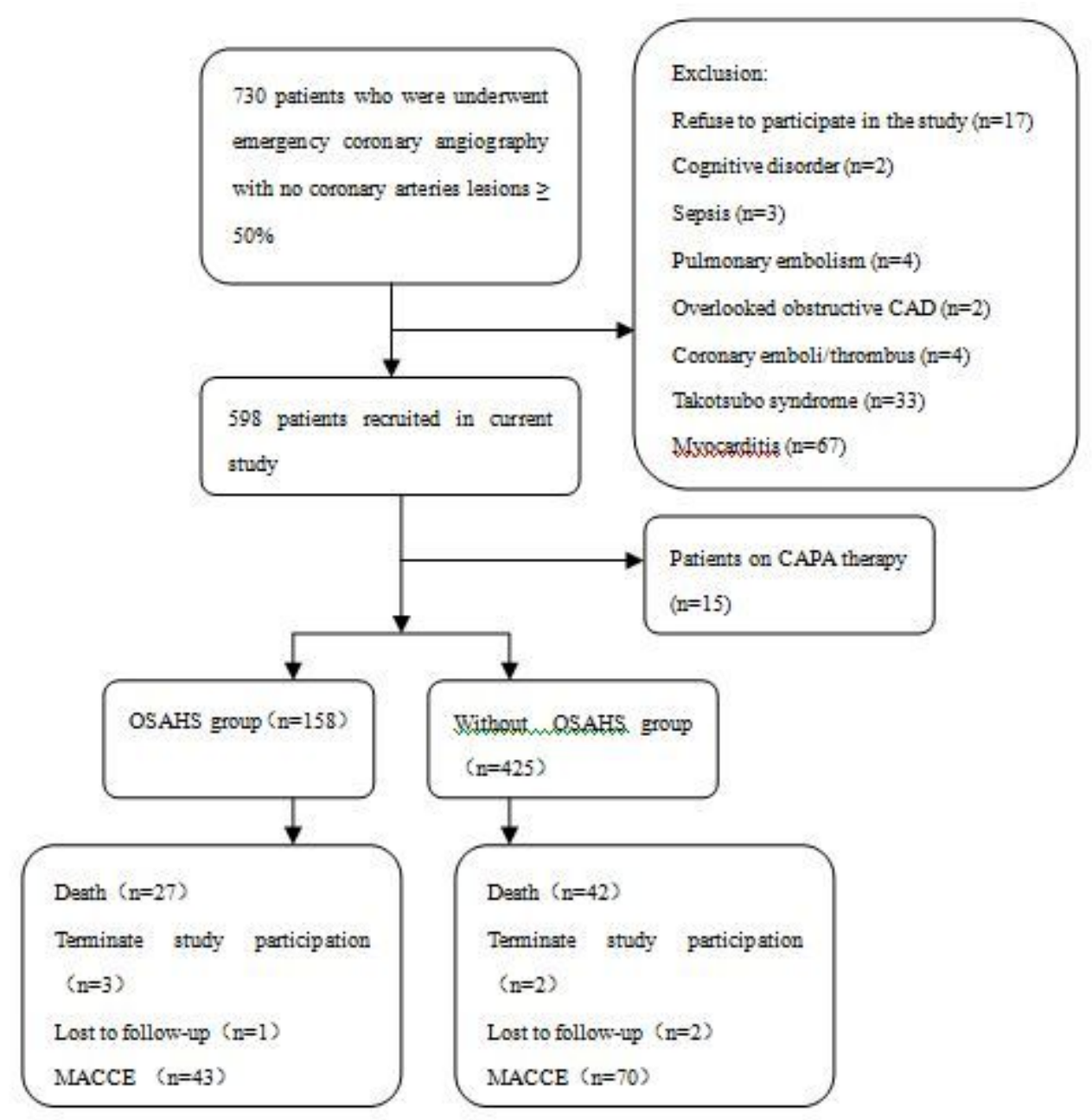

\section{Figure 1}

Flowchart of the selection process and dropouts of the current study 


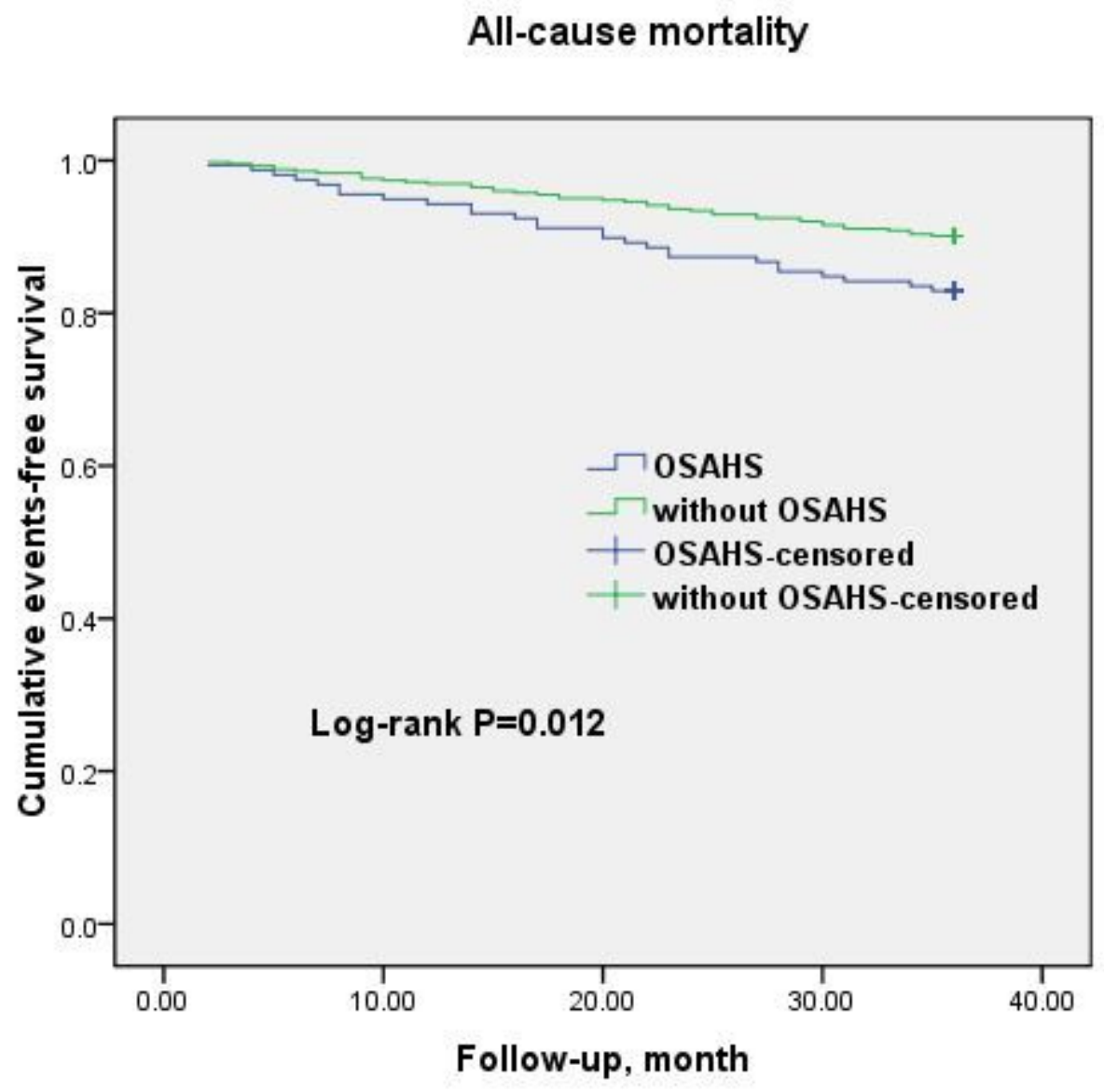

Figure 2

Cumulative event-free survival (Kaplan-Meier curves) for all-cause mortality in OSAHS patients compared to patients without OSAHS 
Major adverse cardiovascular or cerebrovascular events

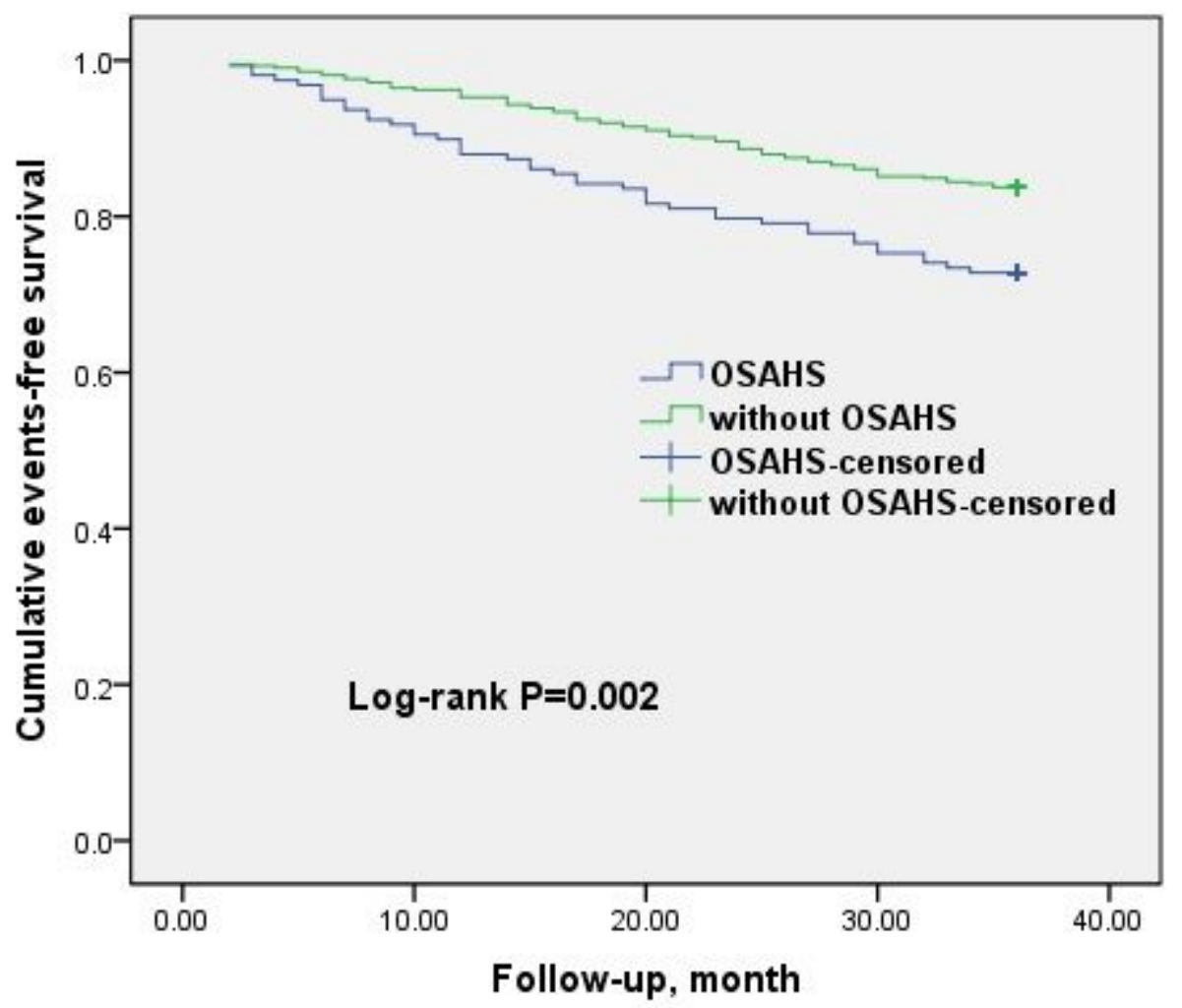

Figure 3

Cumulative event-free survival (Kaplan-Meier curves) for MACCE in OSAHS patients compared to patients without OSAHS. 